\title{
Infantile systemic hyalinosis
}

INSERM

\section{Source}

INSERM. (1999). Orphanet: an online rare disease and orphan drug data base. Infantile systemic hyalinosis. ORPHA:2176

Infantile systemic hyalinosis (ISH) is a very rare disorder belonging to the heterogeneous group of genetic fibromatoses and is characterized by progressive joint contractures, skin abnormalities, severe chronic pain and widespread deposition of hyaline material in many tissues such as the skin, skeletal muscle, cardiac muscle, gastrointestinal tract, lymph nodes, spleen, thyroid, and adrenal glands. 